\title{
Single photon emission computed tomography in patients with acute hydrocephalus or with cerebral ischaemia after subarachnoid haemorrhage
}

\author{
Djo Hasan, Jacob van Peski, Ina Loeve, Eric P Krenning, Marinus Vermeulen
}

\begin{abstract}
Using single photon emission computed tomography (SPECT), cerebral blood flow was studied in eight patients with gradual deterioration in the level of consciousness after subarachnoid haemorrhage. Four had cerebral ischaemia and four had acute hydrocephalus. In patients with cerebral ischaemia, single photon emission computed tomography scanning showed multiple regions with decreased uptake of technetium-99M labelled $d, l$-hexamethyl-propylene amine oxime $\left({ }^{99 m} \mathrm{Tc}\right.$ HM-PAO) mainly in watershed areas. In patients with acute hydrocephalus, decreased uptake was seen mainly in the basal parts of the brain: around the third ventricle, around the temporal horns of the lateral ventricles, and in the basal part of the frontal lobe. After serial lumbar puncture, there was improvement of the uptake of ${ }^{99 \mathrm{~m}}$ Tc HM-PAO in these basal areas in three (convincingly in two and slightly in the other) of the four patients accompanied by clinical improvement in these three patients. These results suggest that patients with acute hydrocephalus and impaired consciousness after SAH, in contrast to patients with cerebral ischaemia, have decreased cerebral blood flow predominantly in the basal parts of the brain.
\end{abstract}

University Hospital Dijkzigt, Rotterdam, The Netherlands Department of

Neurology

D Hasan

$M$ Vermeulen

Department of

Nuclear Medicine

$\mathrm{J}$ van Peski

I Loeve

E P Krenning

Correspondence to:

Dr Hasan, Department of Neurology, University Hospital Dijkzigt Rotterdam 40 Dr Molewaterplein, 3015 GD Rotterdam, The Netherlands

Received 29 June 1989 and in final revised form 27 April 1990.

Accepted 18 May 1990 changes in the regional cerebral blood flow was introduced, by means of intravenously
Recently, a new method to invest administered technetium-99M labelled $d, l$ hexamethyl-propylene amine oxime $\left({ }^{99 \mathrm{~m}} \mathrm{Tc}\right.$ HM-PAO) and by the measurement of its regional cerebral uptake by single photon emission computed tomography (SPECT) ${ }^{11}$

The aim of this study was to compare the pattern of cerebral blood flow, as depicted by SPECT scanning and ${ }^{99 \mathrm{~m}} \mathrm{Tc}$ HM-PAO, in patients with acute hydrocephalus with those with cerebral ischaemia after subarachnoid haemorrhage.

\section{Patients and methods}

We studied eight patients with subarachnoid haemorrhage, four of them with delayed cerebral ischaemia, and four with acute hydrocephalus. All patients had suffered a deterioration of consciousness after admission, defined as a sum score of 12 or less on the 14 point Glasgow Coma Scale. ${ }^{12} 13$ Aneurysmal subarachnoid haemorrhage was confirmed by CT scanning on admission (initial CT). ${ }^{14}$ SPECT studies were performed when cerebral ischaemia or hydrocephalus was suspected to be the cause of the deterioration. Cerebral ischaemia was defined as a deterioration of consciousness with or without focal neurological signs, and without hydrocephalus or an enlarging haematoma on CT. Continuing deterioration immediately after admission was not regarded as cerebral ischaemia. Acute hydrocephalus was diagnosed if the bicaudate index, which was measured on the initial CT or on a repeat CT within one week after the initial SAH, exceeded the normal upper limit $\left(95^{\text {th }}\right.$ percentile) for age,,$^{341516}$ and if deterioration of consciousness occurred with no detectable cause other than hydrocephalus.

Technetium labelled HM-PAO is prepared by adding 1110 megabecquerel freshly eluted sodium pertechnetate to a vial containing freeze dried $0.5 \mathrm{mg} d, l$-hexamethyl-propylene amine oxime (HM-PAO), 7.6 microgram stannous chloride dihydrate, and $4.5 \mathrm{mg}$ sodium chloride (Ceretec). From this mixture, 740 megabecquerel was intravenously administered within 30 minutes after preparation. SPECT scanning was performed 10 to 20 minutes later to avoid possible interference from cerebral uptake of free sodium pertechnetate. Data acquisition was done by means of a single-head rotating gamma-camera (Siemens) with a low energy all purpose collimator and a PDP 11/73 computer. Software: SPETS-11 running under TSX+, Nuclear Diagnostics. Total acquisition angle: 


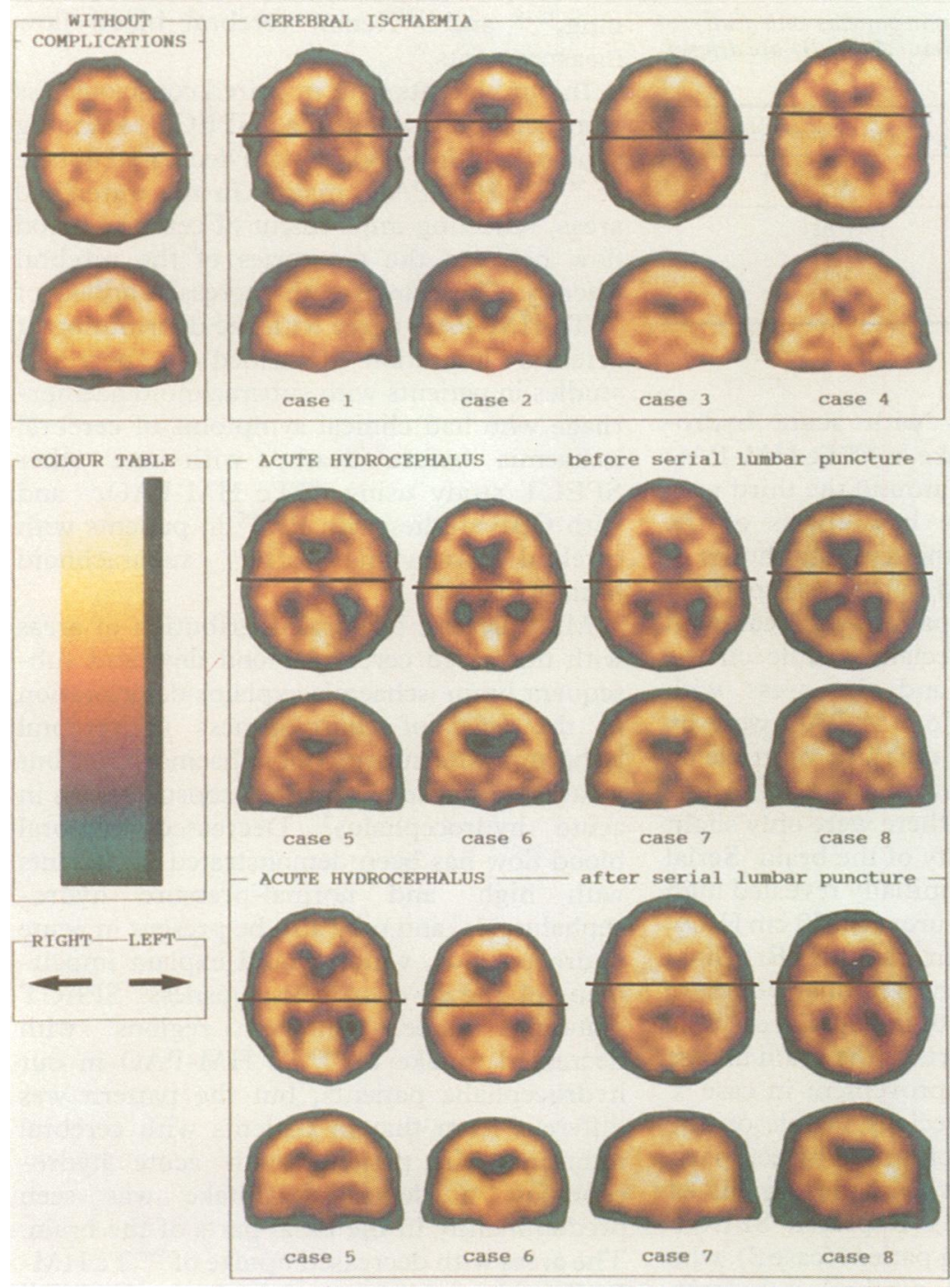

Figure 1 Results of single photon emission computed tomography in patients with cerebral ischaemia and in those with acute hydrocephalus after subarachnoid haemorrhage. Horizontal lines on the transversal slices indicate the corresponding coronal slices.

Key to fig 1

Without complications

SPECT scan of cerebral uptake of ${ }^{99 \mathrm{~m}} \mathrm{Tc} \mathrm{HM}-\mathrm{PAO}$ in a patient with subarachnoid

haemorrhage, who has no complication.

\section{Cerebral ischaemia}

Areas with decreased cerebral uptake of ${ }^{99 \mathrm{~m}} \mathrm{Tc} \mathrm{HM}-\mathrm{PAO}$ in patients with cerebral ischaemia after subarachnoid haemorrhage (coronal and transversal slices):

Case 1: left middle cerebral artery and left anterior and left posterior watershed areas.

Case 2: in the territory of the right middle cerebral artery, right anterior and both posterior watershed areas.

Case 3: right middle cerebral artery and both posterior watershed areas

Case 4: left and right middle cerebral artery and anterior cerebral artery, right anterior watershed area.

Acute hydrocephalus, before serial lumbar puncture

Areas with decreased cerebral uptake of ${ }^{99 \mathrm{~m}} \mathrm{Tc} \mathrm{HM}-\mathrm{PAO}$ in patients with acute

hydrocephalus after subarachnoid haemorrhage, before serial lumbar puncture Case 5, 6, 7 and 8 :

transversal slices: around the ventricles, especially around the posterior horn of the lateral ventricles. Case 5: diffusely distributed areas with decreased uptake of ${ }^{99 m} \mathrm{~T} c$ HM-PAO

coronal slices: around the third ventricle and the temporal horn of the lateral ventricles in the basal parts of the brain. Case 7: a small area on the left side of the lateral ventricle.

Acute hydrocephalus, after serial lumbar puncture

Areas with decreased cerebral uptake of ${ }^{99 \mathrm{~m}} \mathrm{Tc}$ HM-PAO in patients with acute hydrocephalus after subarachnoid haemorrhage, after serial lumbar puncture Case 5, 6 and 7 (coronal slices):

changed pattern of ${ }^{99 m}$ Tc HM-PAO uptake in the basal parts of the brain (slightly in Case 5, convincingly in Case 6 and 7).

Case 8 (transversal and coronal slices)

new multifocal areas with decreased cerebral uptake of ${ }^{99 \mathrm{~m}} \mathrm{Tc} \mathrm{HM}-\mathrm{PAO}$, in the territory of the right middle cerebral artery, in the right and left posterior-, and in the right anterior-watershed areas. $360^{\circ} ; 60$ projections of 30 seconds each; $64 \times 64$ matrix; pixel size: $6 \mathrm{~mm}$. Spatial resolution for tomographic imaging $(12.42 \mathrm{~mm})$ was estimated by the calculation of the full width half maximum for ${ }^{99 \mathrm{~m}} \mathrm{Tc}$ using an air distance between the object and the rotating collimator of $15 \mathrm{~cm}$. Acquired images were filtered by means of a Wiener filter (EMTFLT version $4 \cdot 1$, by Sten Carlsson, Department of Medical Physics, S451-80 Udevalla, Sweden). Transversal (parallel to orbito-meatal line), and coronal slices were reconstructed with a ramp filter, after correction for attenuation, by calculation of the geometric means. Slice thickness: $12 \mathrm{~mm}$. The upper threshold was kept at $100 \%$ and the lower at $5 \%$. The reconstructed images were studied in the multi-slice, absolute scaling mode.

\section{Results}

Patients

Cerebral ischaemia was diagnosed in four patients (cases 1-4). Deterioration from ischaemia occurred between day 3 and day 9 after subarachnoid haemorrhage. All had focal neurological signs: one patient had a right hemiparesis and aphasia (case 1); case 2 had paresis of the left arm; case 3 had a left hemiparesis; and the fourth patient (case 4) had a paralysis of the left leg and a right hemiparesis. None of the four patients had an impaired level of consciousness before deterioration from cerebral ischaemia. A repeat CT failed to show cerebral infarction in two (cases 1 and 3 ) of these four patients. A repeat CT in case 2 showed a hypodense area in the territory of the right middle cerebral artery and in case 4 repeat CT showed a hypodense area in the territory of the left anterior cerebral artery. Both hypodensities correlates with one of the areas with decreased cerebral uptake of ${ }^{99 \mathrm{~m}} \mathrm{Tc}$ $\mathrm{HM}-\mathrm{PAO}$ (fig 1) and with the focal neurological deficits of these two patients.

Another four patients deteriorated from hydrocephalus (cases 5-8). The relative bicaudate indexes in these patients were: 1.30 , $1 \cdot 30,1.05$, and 1.41 . Deterioration from acute hydrocephalus occurred between day 1 and day 4 after subarachnoid haemorrhage. After serial lumbar puncture, three of the four patients showed improvement of the level of consciousness. The remaining patient (case 8 ) developed cerebral ischaemia a few days after the onset of deterioration from acute hydrocephalus.

\section{Spect Studies}

In patients with cerebral ischaemia, multiple circumscribed areas with decreased uptake of ${ }^{99 m}$ T c HM-PAO were seen on the SPECT scan. The posterior watershed areas were involved in all patients and the anterior watershed areas in three (cases 1, 2, and 4). Three patients (cases 2, 3 , and 4) had bilateral areas with decreased uptake of ${ }^{99 \mathrm{~m}} \mathrm{Tc}$ HM-PAO (fig 1). The average counts/cell/minute of the patient without complications and of case $1,2,3$ and 4 in the slices presented in fig 1 were not different (1002/cell/ $\mathrm{min}, 1074 / \mathrm{cell} / \mathrm{min}, 1174 / \mathrm{cell} / \mathrm{min}, 970 /$ cell $/$ $\mathrm{min}$, and $906 /$ cell $/ \mathrm{min}$ respectively). 
Table Relative average counts in patients with acute hydrocephalus. Regions of interest ( $A$ and $B$ ) are defined in fig 2

\begin{tabular}{lll}
\hline & \multicolumn{2}{c}{ Relative average counts (region A/region B) } \\
\cline { 2 - 3 } & before $L P^{\star}$ & after $L P^{\star}$ \\
\hline Case 5 & 0.75 & 0.82 \\
Case 6 & 0.78 & 0.98 \\
Case 7 & 0.87 & 1.01 \\
Case 8 & 0.79 & 0.80 \\
\hline${ }^{\star} L P=$ lumbar puncture. &
\end{tabular}

In the four patients with acute hydrocephalus, decreased uptake of ${ }^{99 \mathrm{~m}}$ Tc HM-PAO was seen predominantly around the third ventricle, bilaterally in the basal parts of the temporal lobes, around the temporal horns of the lateral ventricles, and in the basal parts of the frontal lobes. This pattern of areas with decreased uptake is especially visible on the coronal slices (fig 1) and the areas with decreased uptake of ${ }^{99 \mathrm{~m}} \mathrm{Tc} \mathrm{HM}-\mathrm{PAO}$ were by far larger than the size of the third ventricle on CT $(12 \mathrm{~mm}, 10 \mathrm{~mm}, 8 \mathrm{~mm}$, and $11 \mathrm{~mm}$ respectively). In these patients there were only slight flow voids at the convexity of the brain. Serial lumbar puncture, which initially revealed high cerebro-spinal fluid pressures $\left(40-50 \mathrm{~cm} \mathrm{H}_{2} \mathrm{O}\right)$, was performed in all four patients. Repeated SPECT scanning after serial lumbar puncture showed an improvement of the uptake of ${ }^{99 \mathrm{~m}} \mathrm{Tc}$ $\mathrm{HM}-\mathrm{PAO}$ in the basal parts of the brain in case 6 and 7, and a slight improvement in case 5. These changes were especially visible on the coronal slices and were confirmed by comparison of relative counts within the regions of interest (table and fig 2). Repeated SPECT scanning of the remaining patient (case 8), who developed symptoms of cerebral ischaemia, showed decreased uptake of ${ }^{99 \mathrm{~m}} \mathrm{Tc}$ HM-PAO in both posterior watershed areas, predominantly on the left side, in the territory of the right middle cerebral artery, and in the right anterior watershed area.

\section{Discussion}

Several studies have shown that cerebral uptake of ${ }^{99 \mathrm{~m}} \mathrm{Tc}$ HM-PAO reflects cerebral blood flow. ${ }^{117}$ Decreased cerebral uptake of ${ }^{99 \mathrm{~m}} \mathrm{Tc} \mathrm{HM}-\mathrm{PAO}$ in cerebro-vascular disease has been shown to correspond well with the results of other methods such as PET scan-

\footnotetext{
Figure 2 Regions of interest in patients with acute hydrocephalus. The average relative counts were defined as average counts per cell per minute of region $A$ divided by that of region $B$.
}

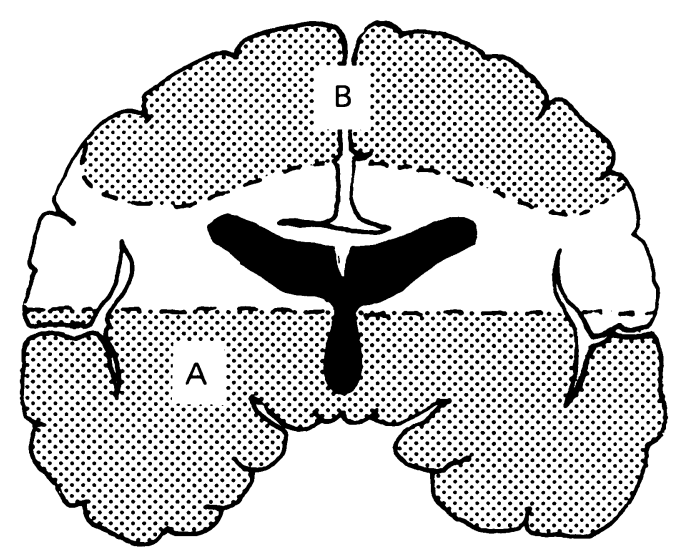

ning, ${ }^{1819}$ and ${ }^{133}$ Xenon cerebral blood flow measurements. ${ }^{20}$

In our patients with impaired consciousness from cerebral ischaemia, SPECT scanning showed multiple regions with decreased uptake of ${ }^{99 \mathrm{~m}} \mathrm{Tc}$ HM-PAO, mainly in the watershed areas, reflecting impairment of cerebral blood flow between the territories of the cerebral arteries. These areas with decreased uptake of ${ }^{99 m}$ Tc HM-PAO agree with the distribution of cerebral infarction described in necropsy studies in patients with subarachnoid haemorrhage who had clinical symptoms of cerebral ischaemia before death, ${ }^{1}$ with one other SPECT study using ${ }^{99 m}$ Tc HM-PAO, ${ }^{21}$ and with CBF studies with PET $^{2}$ in patients with cerebral ischaemia after subarachnoid haemorrhage.

Multiple and bilateral distribution of areas with decreased cerebral blood flow and subsequent brain ischaemia explains deterioration in the level of consciousness in cerebral ischaemia after subarachnoid haemorrhage but what is the cause of impaired consciousness in acute hydrocephalus? Decreased cerebral blood flow has been demonstrated in patients with high $^{9}$ and normal-pressure hydrocephalus, ${ }^{5-810}$ and may also be present in acute hydrocephalus, which would explain impairment of the level of consciousness. SPECT scanning indeed showed regions with decreased uptake of ${ }^{99 \mathrm{~m}} \mathrm{Tc} \mathrm{HM}-\mathrm{PAO}$ in our hydrocephalic patients, but the pattern was different from that in patients with cerebral ischaemia. In patients with acute hydrocephalus, a decreased uptake was seen predominantly in the basal parts of the brain. The areas with decreased uptake of ${ }^{99 \mathrm{~m}} \mathrm{Tc} \mathrm{HM}$ PAO in the basal parts of the brain improved convincingly in two patients (case 6 and 7) and slightly in another patient (case 5), when compared with the higher parts of the brain, after treatment with serial lumbar puncture. This was accompanied by clinical improvement in these three patients. The patient who did not improve had developed cerebral ischaemia.

Decreased radio-activity in the basal areas in patients with acute hydrocephalus cannot be explained by compression of brain tissue by the enlarged ventricles without reduction of the regional blood flow, because in that case each unit of volume of the compressed brain tissue would have shown a higher radio-activity on the SPECT scan. Moreover, brain tissue does not compress but shifts outward and upward displacing CSF. The decreased uptake cannot be explained by a partial volume phenomenon from the third ventricle because the area of decreased uptake is far larger than that of the third ventricle on CT. The decreased uptake most likely reflects decreased cerebral blood flow, but the question is whether there is a primarily decreased cerebral blood flow or a decreased blood flow secondary to hypometabolism. A primary disturbance of cerebral blood flow is the most likely explanation since it was shown in patients with recentonset obstructive hydrocephalus associated with cerebral neoplasia that the levels of cortical blood flow were inappropriately low 
compared with the levels of cortical oxygen utilisation. ${ }^{22}$ The pattern of regional cerebral blood flow impairment that we found in patients with hydrocephalus has not been reported before. In most studies, ${ }^{589}$ however, non rotating, multiple collimated scintillation detectors were used, and cerebral blood flow values of the grey matter were estimated by calculating the initial slope index of the ${ }^{133}$ Xenon clearance curve ${ }^{23} 24$ or by analysing the grey matter part of the bicompartmental model. ${ }^{2324}$ Obviously changes in the blood flow to the deep regions of the brain cannot be detected with this method. Others measured regional cerebral blood flow by means of SPECT and ${ }^{133}$ Xenon, ${ }^{710}$ but calculated regional cerebral blood flow from slices of at least $5 \mathrm{~cm}$ above the orbito-meatal line. These slices are well above the areas of decreased regional cerebral blood flow found in our patients.

Apart from showing that impaired consciousness in acute hydrocephalus results from a disturbance predominantly in the basal parts of the brain, this study may also have practical implications. If a patient has deteriorated from acute hydrocephalus and does not respond to treatment, the explanation may be that insufficient cerebro-spinal fluid drainage or another complication such as cerebral ischaemia has developed. The latter is most likely if SPECT scanning shows decreased regional cerebral blood flow elsewhere than in the basal parts of the brain, even if CT shows no evidence of infarction.

We are grateful to Professor Jan van Gijn for his comments, to Mrs Betty Mast for her excellent secretarial help, and to Ambroos Reijs for technical assistance.

1 Hijdra A, van Gijn J, Stefanko S, van Dongen KJ, Vermeulen $M$, van Crevel H. Delayed cerebral ischemia fter aneurysmal subarachnoid hemorrhage. Clinicoanatomic correlations. Neurology 1986;36:329-33.

2 Powers WJ, Grubb RL, Baker RP, Mintun MA, Raichle ME. Regional cerebral blood flow and metabolism in reversible ischemia due to vasospasm. Determination by positron emission tomography. $J$ Neurosurg 1985;62: p39-46.

3 Van Gijn J, Hijdra A, Wijdicks EFM, Vermeulen M, van Crevel $\mathrm{H}$. Acute hydrocephalus after aneurysmal subCrevel $H$. Acute hydrocephalus after aneurysmal

4 Hasan D, Vermeulen M, Wijdicks EFM, Hijdra A, van Gijn
J. Management problems in acute hydrocephalus after subarachnoid hemorrhage. Stroke 1989;20:747-53.

5 Mamo HL, Philippe CM, Ponsin JC, Rey AC, Luft AG Seylaz JA. Cerebral blood flow in normal pressure hydrocephalus. Stroke 1987;18:1074-80.

6 Menon BW, Weir B, Overton T. Ventricular size and cerebral blood flow following subarachnoid hemorrhage. J Comput Assist Tomogr 1981;5:328-33.

7 Vorstrup S, Christensen J, Gjerris F, Sorensen S, Thomsen AM, Paulson OB. Cerebral blood flow in patients with AM, Paulson OB. Cerebral blood flow in patients with normal pressure hydrocephal

8 Tamaki N, Kusunoki T, Wakabayashi T, Satoshi $M$ Cerebral hemodynamics in normal pressure hydrodynamic computed tomography study. J Neurosurg 1984;61:510-14.

9 Hayashi M, Kobayashi H, Kawano H, Yamamoto S, Maeda $T$. Cerebral blood flow and intracranial pressure patterns in patients with communicating hydrocephalus after subarachnoid hemorrhage. J Neurosurg 1984;61:30-6.

10 Graff-Radford NR, Rezai K, Goderski JC, Eslinger P, Damasio H, Kirchner P. Regional cerebral blood flow in normal pressure hydrocephalus. J Neurol Neurosurg Psychiatry 1987;50:1589-96.

11 Neirinckx RD, Canning LR, Piper IM, et al. Technetium99M d,l-HM-PAO: a new radiopharmaceutical for SPECT imaging of regional cerebral blood perfusion. $J$ Nucl Med 1987;28:191-202.

12 Teasdale GM, Jennett B. Assessment of coma and impaired consciousness. A practical scale. Lancet 1974;ii:81-4.

13 Lindsay KW, Teasdale GM, Knill-Jones RP. Observer variability in assessing the clinical features of subarachnoid hemorrhage. J Neurosurg 1983;58:57-62.

14 Van Gijn J, Van Dongen KJ. Computed tomography in subarachnoid hemorrhage, difference between patients with and without an aneurysm on angiography. Neurology 1980;30:538-9.

15 Earnest MP, Heaton RK, Wilkinson WE, Manke WF. Cortical atrophy, ventricular enlargement and intellectua impairment in the aged. Neurology 1979;29:1138-43.

16 Meese $W$, Kluge W, Grumme T, Hopfenmüller W. Computed tomography evaluation of the cerebro-spinal fluid
spaces of healthy persons. Neuroradiology $1980 ; 19: 131-6$.

spaces of healthy persons. Neuroradiology 1980;19:131-6.
17 Lear JL. Quantitative local cerebral blood flow measurement with technetium-99M HM-PAO: evaluation using multiple radionuclide digital quantitative autoradiography. J Nucl Med 1988;29:1387-92

18 Nishizawa S, Yonekura Y, Fujita T, et al. Brain perfusion single photon emission computed tomography with ${ }^{99 \mathrm{~m}} \mathrm{~T}$ HM-PAO: comparative study with I-123 IMP and cerebral blood flow measured by positron emission tomography (Abstract). J Nucl Med 1987;28:569.

19 Yonekura Y, Nishizawa S, Mukai T, et al. SPECT with ${ }_{99 m}$ Tc- $d, l$-hexamethyl-propylene amine oxime (HMPAO) compared with regional cerebral blood flow measured by PET. Effects of linearisation. J Cereb Blood Flow 1988;8:S82-S89.

20 Andersen AR, Friberg H, Lassen NA, Kristensen $K$, Neirinckx RD. Serial studies on cerebral blood flow using Neirinckx RD. Serial studies on cerebral blood flow using Comm 1987;8:549-57.

21 Davis S, Andrews J, Lichtenstein M, et al. A single-photon emission computed tomography study of hypoperfusion after subarachnoid hemorrhage. Stroke 1990;21:252-9.

22 Brooks DJ, Beaney RP, Powell M, et al. Studies on cerebral oxygen metabolism, blood flow, and blood volume in patients with hydrocephalus before and after decompression, using positron emission tomography. Brain 1986;109:613-28.

23 Lassen NA. Cerebral blood flow measured by xenon-133. Nucl Med Comm 1987;8:535-48.

24 Geraud G, Tremoulet M, Guell A, Bes A. The prognostic value of noninvasive cerebral blood flow measurement in vabarachnoid hemorrhage. Stroke 1984;15:301-5. 